\title{
A Combinational Buffer Management Scheme in Mobile Opportunistic Network
}

\author{
Soujanya Ambala \\ M.Tech (Software Engineering), \\ Assistant Professor, Siddhartha \\ Institute of Technology \& \\ Sciences, Hyderabad, \\ Telangana, India
}

\author{
Dr. Srinivas Ambala \\ M.Tech (CSE), Associate \\ Professor, Vignan's Institute of \\ Management and Technology for \\ Women, Ghatkesar, Telangana, \\ India
}

\author{
Sreedhar Ambala \\ M.Tech (CSE), Assistant Professor \\ Siddhartha Institute of Technology \\ \& Sciences, Hyderabad, \\ Telangana, India
}

\begin{abstract}
Nodes in Mobile Opportunistic Network (MON) have to cache packets to deal with the intermittent connection. The buffer management strategy obviously impacts the performance of MON, and it attracts more attention recently. Due to the limited storage capacity of nodes, traditional buffer management strategies just drop messages based on the property of message, and they neglect the collaboration between neighbors, resulting in an ineffective performance improvement. Therefore, effective buffer management strategies are necessary to ensure that each node has enough buffer space to store the message when the node buffer is close to congestion. In this paper, we propose a buffer management strategy by integrating the characteristics of messages and nodes, and migrate the redundant messages to the neighbor to optimize the total utility, instead of deleting them. The simulation experiment results show that it can obviously improve the delivery ratio, the overhead ratio and the average delays, and reduce the amount of hops compared with the traditional ones.
\end{abstract}

KEYWORDS: mobile opportunistic network; buffer management; node utility; message migration

\section{Introduction}

Mobile Opportunistic Network (MON) [1] is a special kind of ad hoc network that uses the node mobility to transfer packets and becomes more popular in recent years. With the rapid development of wireless communication technology, as well as the widespread popularity of communication equipment, the applications of MON are extended significantly from disaster recovery networks [2], wildlife tracking networks [3], and interplanetary networks [4] to mobile vehicle networks [5]. However, due to intermittent connection and unstable links between nodes, it adopts a storing-carrying-forwarding paradigm to communicate and forward messages through a hop-by-hop style. Connections between nodes are sparse and the contact time between nodes is intermittent. The unpredictability of network links requires nodes to cache messages until they meet the destination node. Nodes exchange information with each other when they contact, and forward messages based on flooding to increase their delivery ratio. The excessive duplicate copies in the network results in the node buffer congestion severely, which leads to the decline of the utilization of the network resource. Therefore, buffer management strategies play an important role in the transmission of the opportunistic network, and they have a direct impact on the message delivery ratio, overhead ratio and average delivery delay, etc. It is important to design an efficient buffer management strategy in MON. The existing research of buffer management in opportunistic network mainly include the following strategies: Drop Head (DH), Drop Tail (DT), Drop Random (DR), Remaining Lifetime (RL), Drop Most Forwarded (MOFO), Least Recently Received (DLRR), and history-based drop [6,7]. The above buffer management strategies usually sort the messages by considering the property of messages [8]

\section{Buffer Management}

The design of the buffer management strategy should be combined with the attributes of the node and the attributes of the message. Most studies only consider 


\section{www.ijtsrd.com}

the properties of the message and ignore those of nodes. In addition, since the motion trajectory of the node depends on the mobility model, the attributes of the nodes are significantly different. We propose a combinational buffer management scheme by collecting and analyzing the state of the message and considering the node properties in mobile opportunistic network. We describe it from four aspects: preliminaries, queuing strategy, utility value calculation and evaluation method, respectively. We give the details in the following sections.

\section{Preliminaries}

Mobile nodes change their locations over time in MONs. In general, evaluating the performance of the routing algorithm is based on the movement trajectories and paths formed by the motion of the nodes. In this paper, we use a real trace, Korea Advanced Institute of Science and Technology (KAIST) and a new mobility model, Self-Similar
Least-Action Human Walk (SLAW). We use a dynamic graph $G=(V, E)$ to simulate the movement model of the nodes in MONs, where $\mathrm{V}$ is a set of nodes and $\mathrm{E}$ is the connection between any two nodes. Nodes exchange information with each other when they are in the communication range. We randomly specify the source node and the destination node in MON. Figure 1 shows the movement trajectory of the node $\mathrm{A}$ to the node $\mathrm{D}$. We assume that the contact time between nodes follows the exponentially distributed. In addition, we assume that there are the following properties in MONs:

$>$ Each node has a limited buffer.

$>$ Mobility of nodes is independent and nodes have different contact rates.

$>$ The links have the same bandwidth.

$>$ A short contact duration or low data rate will not complete the message transmission.

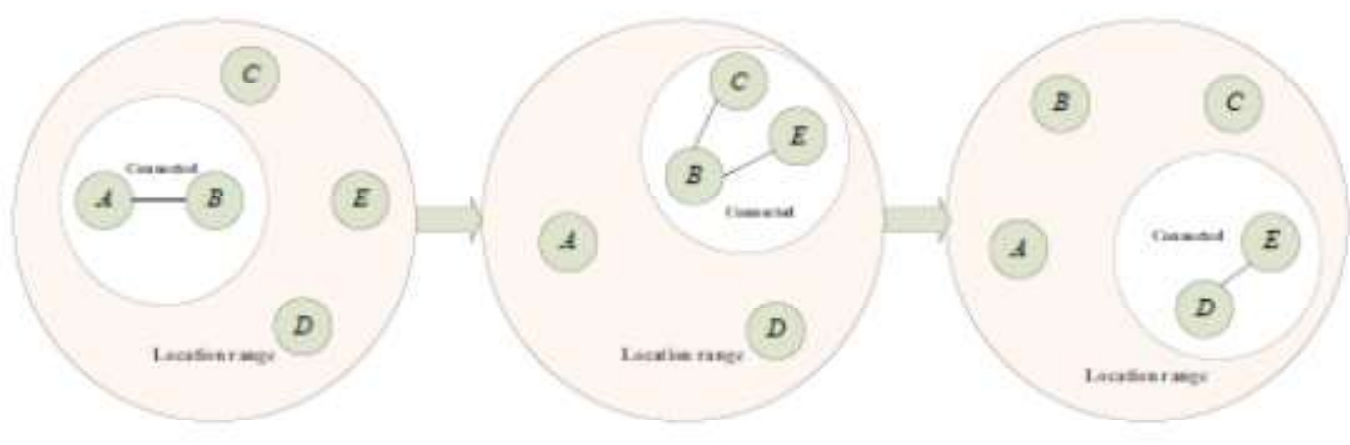

Figure 1. An example of node movement in Mobile Opportunistic Network

\subsection{Queuing Strategy}

The transmission is mainly dependent on the opportunistic communication of nodes in MON. The node is defined as mobile devices that are carried by individuals in general. Therefore, we suppose each message carries a relevant information package $\mathrm{P}=$ $\{$ ui, vi, si, ti, hi\} [17] that contains the message of the source node ui, destination node vi, the size of the message si, the time from the beginning of creation until now ti, and the number of hops from the source node to the current node hi. We set the TTL as the threshold of ti, and delete the message when ti exceeds the TTL. We propose a comprehensive buffer management strategy in mobile opportunistic network.
Figure 2 shows the process of the Combinational Buffer Management (CBM) queuing strategy. We decide that two nodes connect with each other and deliver the message when they enter the communication range of each other. We first sort the priority of the messages in the node before exchanging information. The node $\mathrm{A}$ determines whether it is congested when node B forwards message $i$ to node $A$. If node $A$ has sufficient buffer space, node B directly sends message i to node A, and the node A receives the message $i$ and stores it in the appropriate location in the cache queue as shown in Figure $2 \mathrm{a}$. When the size of the buffer space of node A is not enough to store message $i$, we calculate the utility value of message $i$ and the utility value of node A according to CBM strategy, where the utility value 


\section{www.ijtsrd.com}

of node $\mathrm{A}$ is determined by the average utility value of all messages in the node. If the utility value of message $i$ is less than the utility value of node $A$, the node $\mathrm{A}$ does not store the message $\mathrm{i}$ and the message transfer process failed as shown in Figure $2 \mathrm{~b}$. If the message utility value is higher than the utility value of the node, node A receives the message. However, because the fact that node A does not have enough buffer size, we use the buffer replacement function in the CBM policy to transfer the message with the minimum utility value to the neighbor node $\mathrm{C}$ of the node $\mathrm{A}$ when the node $\mathrm{C}$ has extra available buffer space, (where $\mathrm{q}$ is the message with the lowest utility value in node $A$ ) until the buffer of node $A$ is enough to contain the message [8], as shown in Figure 2c. Then, node $\mathrm{A}$ receives the message $\mathrm{i}$ from node $\mathrm{B}$. When the buffer occupancy rate of the neighbor node $\mathrm{C}$ reaches the threshold, we give up the migration and directly delete the least useful message until node A has enough buffer to store message i. Here, the novelty of our strategy is that we migrate messages that need to delete the neighbor nodes with larger buffers. The proposed migration strategy can effectively assure that the delivery ratio does not decline due to directly deleting the message. The number of message copies is uncontrolled in the opportunistic network. Excessive storage of the same message will inevitably lead to the node buffer space being filled up in a short time; meanwhile, it also causes the waste of network resources, the waste of extensive network resources and the decline of buffer utilization [8]. In order to address this problem, we suppose messages in a node are not redundant. In other words, we determine whether the node keeps the same message when a new message arrives. If there are two messages with the same content, we compare the utility values of the two messages. The advantage of queuing strategy is that it not only avoids the repeated.

\section{Conclusions}

In MONs, transfer information between nodes adopts a storing-carrying-forwarding paradigm due to the node mobility and intermittent connectivity of the network. Node buffer management will directly affect the message delivery ratio due to limited storage space, and rational management strategies will effectively avoid excessive copies of messages and the waste of network resources. The existing buffer management research for opportunistic network is fragmented. In order to solve the present issue, the proposed CBM strategy designs the utility value from the view of messages and nodes. It mainly includes a reasonable queuing strategy and a novel message migration method. The novelty of the strategy is that we proactively forward messages to the neighbor node in order to avoid the reduced delivery ratio of the message caused by deleting messages when there is node buffer congestion. Compared with other classical buffer management strategies, CBM aims to maximize delivery ratio and minimize the number of forwarding hops, overhead ratio and average delays. In the future, we will further study the popularity of the message when calculating the utility value of the message, and use the node position and the node centrality as the criterion of the node utility value. More refined schedule methods will be designed and evaluated. At the same time, we can further verify the correctness and advantages of the CBM strategy by collecting and using more real data sets. At the same time, we should address buffer management in terms of average delay time of messages in future work.

\section{References}

[1] Trifunovic, S.; Kouyoumdjieva, S.T.; Distl, B.; Pajevic, L.; Karlsson, G.; Plattner, B. A Decade of Research in Opportunistic Networks: Challenges, Relevance, and Future Directions. IEEE Commun. Mag. 2017, 55, 168-173.

[2] Ngo, T.; Nishiyama, H.; Kato, N.; Kotabe, S.; Tohjo, H. A Novel Graph-Based Topology Control Cooperative Algorithm for Maximizing Throughput of Disaster Recovery Networks. In Proceedings of the 2016 IEEE 83rd Vehicular Technology Conference (VTC Spring), Nanjing, China, 15-18 May 2016; pp. 1-5.

[3] Dressler, F.; Ripperger, S.; Hierold, M.; Nowak, T. From radio telemetry to ultra-low-power sensor networks: Tracking bats in the wild. IEEE Commun. Mag. 2016, 54, 129-135.

[4] Zhang, L.; Zhou, X.; Guo, J. Noncooperative Dynamic Routing with Bandwidth Constraint in Intermittently Connected Deep Space Information Networks Under Scheduled Contacts. Wirel. Pers. Commun. 2012, 68, 1255-1285.

[5] Santos, R.; Orozco, J.; Ochoa, S.F. A real-time analysis approach in opportunistic networks. ACM SIGBED Rev. 2011, 8, 40-43. 
[6] Boldrini, C. Design and analysis of context-aware forwarding protocols for opportunistic networks. In Proceedings of the Second International Workshop on Mobile Opportunistic Networking, Pisa, Italy, 22-23 February 2010; pp. 201-202.

[7] Pan, D.; Ruan, Z.; Zhou, N.; Liu, X.; Song, Z. A comprehensive-integrated buffer management strategy for opportunistic networks. EURASIP J. Wirel. Commun. Netw. 2013, 2013, 103, doi:10.1186/1687-1499-2013-103.

[8] Erramilli, V.; Crovella, M. Forwarding in opportunistic networks with resource constraints. In Proceedings of the Third ACM Workshop on Challenged Networks, San Francisco, CA, USA, 15 September 2008; pp. 41-48.

[9] Rashid, S.; Ayub, Q.; Zahid, M.S.M.; Abdullah, A.H. Impact of Mobility Models on DLA (Drop Largest) Optimized DTN Epidemic Routing Protocol. Int. J. Comput. Appl. 2011, 18, 35-39.

[10] Kim, D.; Park, H.; Yeom, I. Minimizing the impact of buffer overflow in DTN. In Proceedings of the $3^{\text {rd }}$ International Conference on Future
Internet Technologies (CFI), Seoul, Korea, 18-20 June 2008.

[11] Sati, S.; Probst, C.; Graffi, K. Analysis of Buffer Management Policies for Opportunistic Networks. In Proceedings of the IEEE 25th International Conference on Computer Communication and Networks, Waikoloa, HI, USA, 1-4 August 2016; pp. 1-8.

[12] Krifa, A.; Baraka, C.; Spyropoulos, T. Optimal Buffer Management Policies for Delay Tolerant Networks. In Proceedings of the 5th Annual IEEE Communications Society Conference on Sensor, Mesh and Ad Hoc Communications and Networks, San Francisco, CA, USA, 16-20 June 2008; pp. 260-268.

[13] Scott, K.; Burleigh, S. Bundle Protocol Specification; Internet RFC 5050. Available online: https://rfc-editor.org/ rfc/rfc5050.txt (accessed on 10 November 2017).

[14] Elwhishi, A.; Ho, P.H.; Naik, K.; Shihada, B. A Novel Message Scheduling Framework for Delay Tolerant Networks Routing. IEEE Trans. Parallel Distrib. Syst. 2013, 24, 871-880. 